where

$$
\tan \theta_{1}=\frac{P_{1}{ }^{\prime \ell}-2 \alpha \alpha P}{P_{2}+P\left(\alpha^{2}-\alpha^{2}\right)-\alpha P_{1}}=\tan \left(\alpha t_{1}-\tan ^{-1} \frac{P_{0}^{\prime}}{P_{0}^{\prime \prime}}\right),
$$

and in which $t_{1}$ is the phase difference in seconds between the motion of the sphere and that of the support.

6. The particular class of numbers which are discussed in Mr. Stager's paper suggested themselves in the construction of a table for use in applying Sylow's theorem. This tablenow constructed for the first 10,000 numbers and eventually to be extended to 15,000 or 20,000 - exhibits the value of $k$ for all prime factors of each number which give factors of the form $p(k p+1)$, where $p$ is any prime except 2 and $k$ is any positive integer. Those numbers, called $P$ 's, which contain no factor of the form $p(k p+1)$ form a very interesting class. The present paper deals with their fundamental properties; shows that the numbers consist of four general types ; obtains several formulas for their enumeration; and suggests a connection between the number of ordinary primes and the number of $P$ 's within a given limit.

W. A. Manning, Secretary of the Section.

\title{
THE CONSTRUCTION OF A SPACE FIELD OF EXTREMALS.
}

BY DR. E. GORDON BILL.

(Read before the American Mathematical Society, December 30,1908.)

IT is a well-known theorem of the calculus of variations, ${ }^{*}$ that if all the members of a one parameter family of plane curves pass through a fixed point $O$, then any arc of these extremals which does not contain $O$ nor its conjugate point, may be imbedded in a field.

Moreover, in 1879 Weierstrass stated that a field including $O$ could be constructed and Professor Bliss $†$ has proved this to be true.

* Osgood, Annals of Math., ser. 2, vol. 2, p. 112.

† Bliss, Bulletin, vol. 13, p. 321. 
It is equally true, that if we have a two-parameter family of space curves, through a fixed point $O$, we can construct a space field of extremals including $O$ in its interior.

In order to prove the above statement, we assume $O$ to be an ordinary point of the problem, and choose it for the origin of coordinates.

The extremals * are given by the equations

(1) $x=\phi(s, \alpha, \beta, \gamma), \quad y=\psi(s, \alpha, \beta, \gamma), \quad z=\chi(s, \alpha, \beta, \gamma)$,

where $\phi, \psi, \chi$ are of class $C^{\prime \prime}$ for $s$ in $(0 \bar{S})$ and $\alpha^{2}+\beta^{2}+\gamma^{2}=1$, $s$ being the length of arc measured along an extremal from $O$, and $\alpha, \beta, \gamma$ the direction cosines of the tangents to the extremals at $O$. Under these assumptions the following relations $\dagger$ evidently exist :

$$
\begin{array}{r}
\phi(0)=\psi(0)=\chi(0)=0, \\
\phi_{s}(0)=\alpha, \psi_{s}(0)=\beta, \chi_{s}(0)=\gamma \\
\phi_{s a}(0)=1, \psi_{s \beta}(0)=1, \chi_{s \gamma}(0)=1, \\
\phi_{a}(0)=\phi_{\beta}(0)=\phi_{\gamma}(0)=0, \\
\psi_{a}(0)=\psi_{\beta}(0)=\psi_{\gamma}(0)=0, \\
\chi_{a}(0)=\chi_{\beta}(0)=\chi_{\gamma}(0)=0, \\
\phi_{s \beta}(0)=\phi_{s \gamma}(0)=\psi_{s a}(0)=0, \\
\phi_{s \gamma}(0)=\chi_{s a}(0)=\chi_{s \beta}(0)=0 .
\end{array}
$$

In order to establish the existence of the above field, it will be sufficient to prove the following:

Theonem A. There exists a sphere $S$ having its center at $O$, such that for every point in it $s, \alpha, \beta, \gamma$ are defined as one-valued functions of $x, y, z$ of class $C^{\prime}, i$. e., the equations

$$
\begin{array}{ll}
F_{1} \equiv x-\phi(s, \alpha, \beta, \gamma)=0, & F_{2} \equiv y-\psi(s, \alpha, \beta, \gamma)=0, \\
F_{3} \equiv z-\chi(s, \alpha, \beta, \gamma)=0, & F_{4} \equiv \alpha^{2}+\beta^{2}+\gamma^{2}-1=0,
\end{array}
$$

have one and only one solution $(s, \alpha, \beta, \gamma)$ corresponding to any set of values $(x, y, z)$ in $S$.

Lemma I. There exists a quantity $S^{\prime} \leqq \bar{S}$, such that for $s$ in $\left(0^{*} S^{\prime}\right),\left[0<s \leqq S^{\prime}\right]$, the radius vector $r$ of any point on the extremals is an increasing function of $s$.

* Bliss and Mason, "Properties of curves in space which minimize a definite integral," Transactions Amer. Math. Society, vol. 9, no. 4.

$\dagger \phi(0)=\phi(0, \alpha, \beta, \gamma)$, etc. 

hence

We have $r$ defined as a function of $s$ by $r^{2}=\phi^{2}+\psi^{2}+\chi^{2}$;

$$
\frac{d r}{d s}=\frac{\phi \phi_{s}+\psi \psi_{s}+\chi \chi_{s}}{\sqrt{\phi^{2}+\psi^{2}+\chi^{2}}}
$$

but by Taylor's formulas

$$
\phi=s \phi_{s}\left(\theta_{1}\right), \quad \psi=s \psi_{s}\left(\theta_{2}\right), \quad \chi=s \chi_{s}\left(\theta_{3}\right),
$$

and thus by relations (2)

$$
\lim _{s=0} \frac{d r}{d s}=\frac{\alpha^{2}+\beta^{2}+\gamma^{2}}{\sqrt{\alpha^{2}+\beta^{2}+\gamma^{2}}}=1 .
$$

Hence, from the continuity of $r$, the above $S^{\prime}$ exists and the surface of a sphere of radius

$$
r^{\prime}=\min \sqrt{\phi\left(S^{\prime}\right)^{2}+\psi\left(S^{\prime}\right)^{2}+\chi\left(S^{\prime}\right)^{2}}
$$

will be cut once and only once by each extremal, for a value of $s$ in $\left(0^{*} S^{\prime}\right)$.

Lemma II. There exists a quantity $S^{\prime \prime} \leqq \bar{S}$ such that for $s$ in $\left(0^{*} S^{\prime \prime}\right)$ the Jacobian $\Delta$ of equations (3) does not vanish.

We have

$$
\overline{2}=\left|\begin{array}{cccc}
\phi_{s} & \phi_{a} & \phi_{\beta} & \phi_{\gamma} \\
\psi_{s} & \psi_{a} & \psi_{\beta} & \psi_{\gamma} \\
\chi_{s} & \chi_{a} & \chi_{\beta} & \chi_{\gamma} \\
0 & \alpha & \beta & \gamma
\end{array}\right|
$$

and hence from (2) $\Delta(0, \alpha, \beta, \gamma)=0$; but expanding $\frac{1}{2} \Delta$ in terms of the elements of the last row and applying Taylor's formulas, we have from (2)

$$
\begin{aligned}
\lim _{s=0} \frac{\Delta}{2 s^{2}} & =\alpha\left|\begin{array}{ccc}
\alpha & 0 & 0 \\
\beta & 1 & 0 \\
\gamma & 0 & 1
\end{array}\right|-\beta\left|\begin{array}{lll}
\alpha & 1 & 0 \\
\beta & 0 & 0 \\
\gamma & 0 & 1
\end{array}\right|+\gamma\left|\begin{array}{ccc}
\alpha & 1 & 0 \\
\beta & 0 & 1 \\
\gamma & 0 & 0
\end{array}\right| \\
& =\alpha^{2}+\beta^{2}+\gamma^{2}=1 .
\end{aligned}
$$

Thus in the neighborhood of $O$

$$
\Delta=2 s^{2}[1+E(s)] \quad \text { where } \lim _{s=0} E(s)=0,
$$


hence $S^{\prime \prime}$ exists, and about $O$ there is a sphere with radius

$$
r^{\prime \prime}=\min _{l} \overline{\phi\left(S^{\prime \prime \prime}\right)^{2}+\psi\left(S^{\prime \prime}\right)^{2}+\chi\left(S^{\prime \prime}\right)^{2}},
$$

such that for all points in it, except $O$, the Jacobian of equations (3) is not zero.

Let us choose $r>0$ to be less than $r^{\prime}$ and $r^{\prime \prime}$. We wish to show that the sphere $S$ of radius $r$ about $O$ satisfies the conditions of Theorem $A$.

We are assured by Lemma $I$ that for $s$ in a restricted interval, the surface of $S$ is cut once and only once by each extremal through $O$, and by Lemma II we know that for each point of the surface of $S$, the Jacobian of (3) does not vanish.

Next, let $P_{0}\left(x_{0}, y_{0}, z_{0}\right)$ be a point where one of the extremals (1) pierces the surface of $S$. The values of $s, \alpha, \beta, \gamma$ corresponding to $x_{0}, y_{0}, z_{0}$ will satisfy (3), and as the functions $F_{i}$ $\left(i_{0}=1, \cdots, 4\right)$ admit first derivatives in the vicinity of $P_{0}$, and as $\Delta\left(P_{0}\right) \neq 0$, we can state by the well known theorem on implicit functions that:

In a certain zone $Z_{0}$ of $S$ about $P_{0}$ there exist one-valued functions $\bar{s}(x, y, z), \bar{\alpha}(x, y, z), \bar{\beta}(x, y, z)$, and $\bar{\gamma}(x, y, z)$ of class $C^{\prime}$ which reduce to $s_{0}, \alpha_{0}, \beta_{0}, \gamma_{0}$ at $\left(x_{0}, y_{0}, z_{0}\right)$ and which identically satisfy (3) in $x, y, z$ in $Z_{0}$.

The remainder of our problem is to extend the domain of definition of these functions into larger and larger zones of $S$ until finally they are defined for the entire sphere $S$.

First we will extend the domain of definition around the base $B_{0}$ of $Z_{0}$.

Take $P_{1}$ on $B_{0}$ (Fig. I); then as above in a sphere $S_{1}^{\prime}$ about $P_{1}$, functions $s, \alpha, \beta, \gamma$ are defined satisfying conditions required and being identical in $Z_{0}$ with $\bar{s}, \bar{\alpha}, \bar{\beta}, \bar{\gamma}$.

Next take $P_{2}$, the intersection of $S_{1}^{\prime}$ and $B_{0}$; then in $S_{2}^{\prime}$ about $P_{2}$, functions $s, \alpha, \beta, \gamma$ are defined, being identical with $\bar{s}, \bar{\alpha}, \bar{\beta}$, $\bar{\gamma}$ in $Z_{0}$ and $S_{1}^{\prime \prime}$.

Thus, if by means of extension spheres $S_{i}^{\prime}$ we can extend the domain of definition of functions $s, \alpha, \beta, \gamma$ completely around $B_{0}$, we are assured of coming back to $P_{1}$, with the values $s_{1}, \alpha_{1}$, $\beta_{1}, \gamma_{1}$ originally defined there; for each extension sphere $S_{i}^{\prime}$ 
overlaps $Z_{0}$ and $S_{i-1}^{\prime}$ and in these common parts, the functions $s, \alpha, \beta, \gamma$ are identical with those originally defined.

Now, certainly we can extend completely around $B_{0}$ by the above process, provided the lower limit of the radii of the extension spheres $S_{i}^{\prime}$ is not zero. If, however,

$$
\text { lower limit } r_{i}^{\prime}=0 \text {, }
$$

the region of extension will be limited at some point $A$ (Fig. I) on $B_{0}$; but the functions $s, \alpha, \beta, \gamma$ are limited near $A$ and hence must have at least one set of limiting values $(\bar{s}, \bar{\alpha}, \bar{\beta}, \bar{\gamma})$. Moreover, $s, \alpha, \beta, \gamma$ are continuous in $S$ and hence $\bar{s}, \bar{\alpha}, \bar{\beta}, \bar{\gamma}$ are solutions of equations (3), and thus as above we can extend past $(\bar{s}, \bar{\alpha}, \bar{\beta}, \bar{\gamma})$; hence the region of extension is not limited at $A$ and therefore

$$
\text { lower limit } r_{i} \neq 0 \text {. }
$$

Let us now replace all the extension spheres $S_{i}^{\prime}$ by a sphere $S_{m}^{\prime}$ of fixed radius $r_{m}^{\prime}$,

$$
0<r_{m}^{\prime} \leqq \text { lower limit } r_{i}^{\prime} \text {. }
$$

By means of $S_{m}^{\prime}$ we can extend the region of definition of $s, \alpha, \beta, \gamma$ from $Z_{0}$ into $Z_{1}$, a larger zone of $S$.

Similarly we can extend from $Z_{1}$ to $Z_{2}$ to $Z_{3}$, etc., until finally $s(x, y, z), \alpha(x, y, z), \beta(x, y, z)$ and $\gamma(x, y, z)$ are defined for all points of $S$, provided, however, the radii $r_{m}^{i}$ of the uniform extension spheres $S_{m}^{i}$ do not converge to zero.

If

$$
\text { lower limit } r_{m}^{i}=0
$$

the zones $Z_{0}, Z_{1}, Z_{2}$, etc., will be limited in extent by the base $B$ (Fig. I) of a zone $Z$ and beyond this we cannot extend.

But since $B$ is the limiting position of a series of bases $B_{0}$, $B_{1}, B_{2}$, etc., and since $s, \alpha, \beta, \gamma$ remain limited, they must as above approach definite limiting values $(s, \alpha, \beta, \gamma)$ as we approach a point $P$ of $B$.

However $s, \alpha, \beta, \gamma$ are continuous functions in the region considered, hence $(s, \alpha, \beta, \gamma)$ are solutions of equations (3) and thus we can extend past $P, i$. e., past $B$.

So, finally, for every point of $S, s, \alpha, \beta, \gamma$ are defined as onevalued functions of $x, y, z$ of class $C^{\prime}, i$. e., a field has been constructed about $O$.

YALE UNIVERSITY, January 22, 1909. 